International Journal of Pure and Applied Mathematics

Volume 112 No. $4 \quad 2017,741-747$

ISSN: 1311-8080 (printed version); ISSN: 1314-3395 (on-line version)

url: http://www.ijpam.eu

doi: 10.12732 /ijpam.v112i4.6

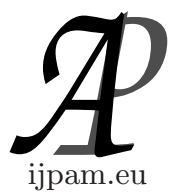

\title{
ON THE MAXIMAL NUMERICAL RANGE OF ELEMENTARY OPERATORS
}

\author{
Flora Mati Runji ${ }^{1}$, John Ogonji Agure ${ }^{2}$, Fredrick Oluoch Nyamwala ${ }^{3}$ \\ ${ }^{1}$ Department of Mathematics, Statistics and Actuarial Sciences \\ Karatina University \\ P.O. Box 1957-10101, Karatina, KENYA \\ ${ }^{2}$ Department of Pure and Applied Mathematics \\ Maseno University \\ P.O. Box 333, Maseno, KENYA \\ ${ }^{3}$ Department of Mathematics and Physics \\ Moi University \\ P.O. Box 3900-30100, Eldoret, KENYA
}

\begin{abstract}
The notion of the numerical range has been generalized in different directions. One such direction, is the maximal numerical range introduced by Stampfli (1970) to derive an identity for the norm of a derivation on $L(H)$. Unlike the other generalizations, the maximal numerical range has not been largely explored by researchers as many only refer to it in their quest to determine the norm of operators. In this paper we establish how the algebraic maximal numerical range of elementary operators is related to the closed convex hull of the maximal numerical range of the implementing operators $A=\left(A_{1}, A_{2}, \ldots, A_{\mathrm{n}}\right)$, $B=\left(B_{1}, B_{2}, \ldots, B_{\mathrm{n}}\right)$, on the algebra of bounded linear operators on a Hilbert space $H$. The results obtained are an extension of the work done by Seddik [2] and Fong [9].
\end{abstract}

AMS Subject Classification: 47A12, 47B47

Key Words: algebraic maximal numerical range, elementary operator

Received: $\quad$ October 24, 2016

Revised: December 8, 2016

Published: February 14, 2017

${ }^{\S}$ Correspondence author (c) 2017 Academic Publications, Ltd. url: www.acadpubl.eu 


\section{Introduction}

For a bounded linear operator $T$ on a Hilbert space $H$, we denote the set of bounded linear operators on $H$ by $L(H)$ and define the numerical range implemented by the operator $T$ by $W(T)=\{\langle T x, x\rangle:\|x\|=1\}$. It is clear $L(H)$ is an algebra when multiplication is pointwise defined. In fact it is a $C^{*}$-algebra with the Hilbert adjoint defining an involution on $L(H)$. For more information on numerical ranges we refer the reader to [3], [4], [5] and [7] and for $C^{*}$-algebra we refer to [14], [15], [16], and [17].

The concept of maximal numerical range was introduced by Stampfli [8] in proving the norm of a derivation.

Definition 1. The maximal numerical range of $T \in L(H)$, denoted by $W_{\circ}(T)$ is the set

$$
W_{\circ}(T)=\left\{\lambda:\left\langle T x_{n}, x_{n}\right\rangle \rightarrow \lambda,\left\|x_{n}\right\|=1,\left\|T x_{n}\right\| \rightarrow\|T\|\right\} .
$$

When $H$ is finite dimensional, $W_{\circ}(T)$ corresponds to the numerical range produced by the maximal vectors (vectors $x$ such that $\|x\|=1$ and $\|T x\|=$ $\|T\|)$.

The Joint maximal numerical range of $A=\left(A_{1}, A_{2}, \ldots, A_{n}\right)$ is given by

$$
W_{\circ}(A)=\left\{\left\{\lambda_{i}\right\} \in \mathbb{C}^{n}:\left\langle A_{i} x_{n}, x_{n}\right\rangle \rightarrow \lambda_{i},\left\|x_{n}\right\|=1,\left\|A_{i} x_{n}\right\| \rightarrow\left\|A_{i}\right\|\right\},
$$

for $1 \leq i \leq n$.

If $\mathscr{A}$ is a $\mathrm{C}^{*}$-algebra with identity $I, a \in \mathscr{A}$ and $\mathscr{A}^{*}$ its dual space, we denote by $S(\mathscr{A})=\left\{f \in \mathscr{A}^{*}: f(I)=1=\|f\|\right\}$, the set of states on $\mathscr{A}$.

The algebraic numerical range of an element $a \in \mathscr{A}$ is the set

$$
V(a ; \mathscr{A})=\{f(a): f \in S(\mathscr{A})\} .
$$

$S_{\circ}(a, \mathscr{A})=\left\{f: f(I)=1=\|f\|, f\left(a^{*} a\right)=\|a\|^{2}\right\}$ is denote the set of maximal states on $\mathscr{A}$. We introduce the following definition:

Definition 2. The algebraic maximal numerical range, denoted by $V_{\circ}(a, \mathscr{A})$ is the set $\left\{f(a): f \in S_{\circ}(a, \mathscr{A})\right\}$

Let $A=\left(A_{1}, A_{2}, \ldots, A_{n}\right), B=\left(B_{1}, B_{2}, \ldots, B_{n}\right)$ be two n-tuples with $A_{i}, B_{i} \in$ $L(H)$ for $1 \leq i \leq n$.

The elementary operator $R_{A, B}$ associated with $A$ and $B$ is the operator on $L(H)$ into itself defined by

$$
R_{A, B}(X)=A_{1} X B_{1}+A_{2} X B_{2}+\cdots+A_{n} X B_{n}, \forall X \in L(H) .
$$

For $T_{1}$ and $T_{2}$ in $L(H)$, we have the following examples of elementary operators: 
i) the left multiplication operator $L_{T_{1}}$ defined by $L_{T_{1}}(X)=T_{1} X, \forall X \in L(H)$;

ii) the right multiplication operator $R_{T_{2}}$ defined by $R_{T_{2}}(X)=X T_{2}, \forall X \in$ $L(H)$;

iii) the elementary multiplication operator $M_{T_{1}, T_{2}}=L_{T_{1}} R_{T_{2}}$ defined by

$$
M_{T_{1}, T_{2}}(X)=T_{1} X T_{2}, \forall X \in L(H),
$$

i.e. the elementary operator of length one;

iv) the inner derivation $\Delta_{T_{1}}$ defined by $\Delta_{T_{1}}(X)=T_{1} X-X T_{1}$;

v) the generalized derivation $\Delta_{T_{1}, T_{2}}$ defined by $\Delta_{T_{1}, T_{2}}(X)=T_{1} X-X T_{2}$.

Symmetric studies on elementary operators begun in the late 1950's with Lumer and Rosenblum [6] establishing their spectral properties and applications to systems of operator equations. More results on their spectral and structural properties are found in, [10], [11], [12], and [13].

If $A$ and $B$ are n-tuples of commuting operators on $H, W(A), W(B)$ the usual numerical ranges of $A$ and $B, V\left(R_{A, B}\right)$ the algebraic numerical range of $R_{A, B}$, then:

(i) [1] establishes that the numerical range of an elementary operator acting on the Banach space of the p-Schatten class operators on $H,\left(\mathscr{C}_{p}(H),\|\cdot\|_{p}\right)$,for $p \geq 1$, satisfies the relation $c o\left(W(A) \circ W(B) \subset V\left(R_{p}(A, B)\right)\right.$;

(ii) in [2] it is proved that

$$
\operatorname{co} \overline{\left\{\sum_{i=1}^{n} \lambda_{i} \beta_{i}\right\}} \subset V\left(R_{A, B}\right),
$$

where $\left(\lambda_{1}, \ldots, \lambda_{n}\right) \in W(A),\left(\beta_{1}, \ldots, \beta_{n}\right) \in W(B)$.

In this paper we establish results using the maximal numerical range.

Notation. For vectors $x$ and $y$ in a Hilbert space $H$, define a finite rank operator by $(x \otimes y) z=\langle z, y\rangle x, \quad \forall z \in H$.

We take $S \subseteq L(H)$ to be an operator algebra containing finite rank operators. For a set $M$, we denote by $\bar{M}$ and $c o M$ the closure and the convex hull of $M$ respectively. 


\section{Main Result}

Theorem 2.1. We have

$$
\operatorname{co} \overline{\left\{\sum_{i=1}^{n} \lambda_{i} \beta_{i}\right\}} \subset V_{\circ}\left(R_{A, B} \mid S\right),
$$

where $\left(\lambda_{1}, \lambda_{2}, \ldots, \lambda_{n}\right) \in W_{\circ}(A),\left(\beta_{1}, \beta_{2}, \ldots, \beta_{n}\right) \in W_{\circ}(B)$ and $V_{\circ}\left(R_{A, B} \mid S\right)$ the algebraic maximal numerical range of the elementary operator restricted on $S$.

Proof. et $\lambda \in W_{\circ}(A), \beta \in W_{\circ}(B)$.

This implies, there exist sequences $\left\{x_{n}\right\},\left\{y_{n}\right\} \in H$ such that $\left\|x_{n}\right\|=\left\|y_{n}\right\|=$ 1 and

$$
\begin{array}{r}
\lim _{n \rightarrow \infty}\left\{\left\langle A_{1} x_{n}, x_{n}\right\rangle, \ldots,\left\langle A_{n} x_{n}, x_{n}\right\rangle\right\}=\lim _{n \rightarrow \infty}\left\{\lambda_{1}, \ldots, \lambda_{n}\right\}=\lambda,\left\|A_{i} x_{n}\right\| \rightarrow\left\|A_{i}\right\|, \\
\lim _{n \rightarrow \infty}\left\{\left\langle B_{1} x_{n}, x_{n}\right\rangle, \ldots,\left\langle B_{n} x_{n}, x_{n}\right\rangle\right\}=\lim _{n \rightarrow \infty}\left\{\beta_{1}, \ldots, \beta_{n}\right\}=\beta,\left\|B_{i} x_{n}\right\| \\
1 \leq i B_{i} \|, \\
1 \leq n .
\end{array}
$$

Define $f$ on $L(S)$ by

$$
f(\Omega)=\lim _{n \rightarrow \infty}\left\langle\Omega\left(x_{n} \otimes y\right) z_{n},\left(x_{n} \otimes y_{n}\right) z_{n}\right\rangle, \quad \forall \Omega \in L(S) .
$$

$f$ is clearly linear and moreover

$$
\begin{aligned}
\left\|x_{n} \otimes y_{n}\right\| & =\sup _{n}\left\{\left\|\left(x_{n} \otimes y_{n}\right) z_{n}\right\|:\left\|z_{n}\right\|=1\right\} \\
& =\sup _{n}\left\{\left|\left\langle z_{n}, y_{n}\right\rangle\right|\left\|x_{n}\right\|:\left\|z_{n}\right\|=1\right\} \\
& =\left\|z_{n}\right\|^{2}\left\|x_{n}\right\| \\
& =1 .
\end{aligned}
$$

Also,

$$
\begin{aligned}
|f(\Omega)| & =\left|\left\langle\Omega\left(x_{n} \otimes y_{n}\right) z_{n},\left(x_{n} \otimes y_{n}\right) z_{n}\right\rangle\right| \\
& =\left|\left\langle z_{n}, y_{n}\right\rangle\right|^{2}\left|\left\langle\Omega x_{n}, x_{n}\right\rangle\right| \\
& \leq\left\|z_{n}\right\|^{2}\left\|y_{n}\right\|^{2}\left\|\Omega x_{n}\right\|\left\|x_{n}\right\| \\
& \leq\left\|z_{n}\right\|^{4}\|\Omega\|\left\|x_{n}\right\|^{2} \\
& =\|\Omega\|, \quad \forall z_{n}=y_{n} .
\end{aligned}
$$


Therefore $f$ is bounded and $\|f\| \leq 1$.

Assume $I \in S$ ( $S$ can be unitized in case its non-unital) then,

$$
\begin{aligned}
f(I) & =\lim \left\langle\left(x_{n} \otimes y_{n}\right) z_{n},\left(x_{n} \otimes y_{n}\right) z_{n}\right\rangle \\
& =\lim \left\langle\left\langle z_{n}, y_{n}\right\rangle x_{n},\left\langle z_{n}, y_{n}\right\rangle x_{n}\right\rangle \\
& =\lim \left|\left\langle z_{n}, y_{n}\right\rangle\right|^{2}\left\|x_{n}\right\|^{2} \\
& =\left\|z_{n}\right\|^{4}\left\|x_{n}\right\|^{2} \\
& =1, \quad \forall z_{n}=y_{n}
\end{aligned}
$$

Hence, $f(I)=1$, so that $\|f(I)\|=1 \leq\|f\| \Longrightarrow\|f\| \geq 1$.

Thus $\|f\|=1$.

A linear functional $f$ is said to be positive if $f\left(\omega \omega^{*}\right) \geq 0$ for all $\omega \in S$. Taking a sequence of unit vectors $z$ in $H$ we see that $f$ is a positive linear functional since

$$
\begin{aligned}
f\left(\Omega \Omega^{*}\right) & =\left\langle\Omega \Omega^{*}\left(x_{n} \otimes y_{n}\right) z_{n},\left(x_{n} \otimes y_{n}\right) z_{n}\right\rangle \\
& =\left\langle\Omega^{*}\left(x_{n} \otimes y_{n}\right) z_{n}, \Omega^{*}\left(x_{n} \otimes y_{n}\right) z_{n}\right\rangle \\
& =\left\|\Omega^{*}\left(x_{n} \otimes y_{n}\right) z_{n}\right\|^{2} \\
& =\left\|\Omega^{*}\right\|^{2} \geq 0 .
\end{aligned}
$$

Since $f$ is a positive linear functional of unit norm, it follows that $f$ is a state on $L(S)$.Moreover, $f$ is clearly a maximal state.

Recall now that

$$
R_{A, B}(X)=\sum_{i=1}^{n} A_{i} X B_{i}=A_{1} X B_{1}+A_{2} X B_{2}+\cdots+A_{n} X B_{n}, \forall X \in L(H)
$$

Thus for $X \in S$ we have,

$$
\begin{aligned}
f\left(\sum_{i=1}^{n} A_{i} X B_{i}\right) & =f\left(\sum_{i=1}^{n} A_{i}\left(x_{n} \otimes y_{n}\right) B_{i} z_{n}\right) \\
& =f\left(\sum_{i=1}^{n} A_{i} x_{n}\left\langle B_{i} z_{n}, y_{n}\right\rangle\right) \\
& =\sum_{i=1}^{n}\left(f\left(A_{i} x_{n}\right)\left\langle B_{i} z_{n}, y_{n}\right\rangle\right) \\
& =\sum_{i=1}^{n} f\left(A_{i} x_{n}\right) g\left(B_{i} y_{n}\right), \quad \forall z_{n}=y_{n}
\end{aligned}
$$




$$
=\sum_{i=1}^{n} \lambda_{i} \beta_{i} \subset V_{\circ}\left(\left.R_{A, B}\right|_{S}\right)
$$

Since the algebraic maximal numerical range is compact and convex, then

$$
\operatorname{co} \overline{\left\{\sum_{i=1}^{n} \lambda_{i} \beta_{i}\right\}} \subset V_{\circ}\left(R_{A, B}\right) .
$$

Corollary 2.2. Let $A \in L(H)$. Then $V_{\circ}\left(L_{A}\right)=V_{\circ}\left(R_{A}\right)=V_{\circ}(A)$.

Proof. If $A$ is an operator on a Hilbert space $H$, Fong [9] has showed that $V_{\circ}(A)=W_{\circ}(A)$. The inclusion $V_{\circ}(A) \subseteq V_{\circ}\left(L_{A}\right)$ follows from this and the above theorem.

Now let $\lambda \in V_{\circ}\left(L_{A}\right)$. Then there exists $f$ in $\left(L\left(L_{A}\right)\right)^{*}$ such that

$$
f\left(L_{A}\right)=\lambda, f(I)=1=\|f\|
$$

and

$$
f\left(L_{A}^{*} L_{A}\right)=\left\|L_{A}\right\|^{2} .
$$

Define a functional $g$ on $L(H)$ by $g(A)=f\left(L_{A}\right)$. By simple computation,we see that $g$ is a maximal state on $L(H)$ so that $g(A)=f\left(L_{A}\right) \in V_{\circ}(A)$. Therefore $V_{\circ}\left(L_{A}\right) \subseteq V_{\circ}(A)$. By the same argument, we find also that $V_{\circ}\left(R_{A}\right)=V_{\circ}(A)$.

Corollary 2.3. For $A, B \in L(H), V_{\circ}(A)-V_{\circ}(B)=V_{\circ}\left(\Delta_{A, B}\right)$.

Proof. By the above theorem, we have $W_{\circ}(A)-W_{\circ}(B) \subseteq V_{\circ}\left(\Delta_{A, B}\right)$ and since $V_{\circ}\left(\Delta_{A, B}\right)$ is closed, then we have

$$
\overline{\left(W_{\circ}(A)-W_{\circ}(B)\right)}=V_{\circ}(A)-V_{\circ}(B) \subseteq V_{\circ}\left(\Delta_{A, B}\right) .
$$

For the reverse inclusion,

$$
\begin{aligned}
V_{\circ}\left(\Delta_{A, B}, \mathscr{A}\right) & =\left\{f\left(\Delta_{A, B}\right): f \in S_{\circ}(L(\mathscr{A}))\right\} \\
& =\left\{f\left(L_{A}-R_{B}\right): f \in S_{\circ}(L(\mathscr{A}))\right\} \\
& \subseteq\left\{f\left(L_{A}\right): f \in S_{\circ}(L(\mathscr{A}))\right\}-\left\{f\left(R_{B}\right): f \in S_{\circ}(L(\mathscr{A}))\right\} \\
& =V_{\circ}\left(L_{A}\right)-V_{\circ}\left(R_{B}\right) \\
& =V_{\circ}(A)-V_{\circ}(B) .
\end{aligned}
$$

Therefore $V_{\circ}\left(\Delta_{A, B}, \mathscr{A}\right) \subseteq V_{\circ}(A)-V_{\circ}(B)$. 


\section{Acknowledgments}

The authors wish to thank National Commission for Science, Technology and Innovation for the financial support of this research work

\section{References}

[1] A. Seddik, The numerical range of elementary operators II, Linear Algebra Appl., 338 (2001), 239-244, doi: 10.1016/S0024-3795(01)00389-5.

[2] A. Seddik, The numerical range of elementary operators, Integr. Eq. Oper. Theory, 43 (2002), 248-252, doi: 10.1007/BF01200256.

[3] F.F. Bonsall and J. Duncan, Numerical Range, Volume I, Cambridge University Press, 1973.

[4] F.F. Bonsall and J. Duncan, Numerical Range, Volume II, Cambridge University Press, 1973.

[5] F.F. Bonsall and J. Duncan, Numerical ranges of operators on normed spaces and elements of normed algebras, London Math. Soc. Lecture Note Series, 2, Cambridge, 1971.

[6] G. Lumer and M. Rosenblum, Linear operator equations, Proc. Amer. Math. Soc., 10 (1959), 32-41, doi: 10.1090/S0002-9939-1959-0104167-0.

[7] K.E. Gustafson and D.K.M. Rao, Numerical Range: The Field of Values of Linear Operators and Matrices, Springer, 1997.

[8] J.G. Stampfli, The norm of a derivation, Pacific J. Math., 33 (1970), 737-747, doi: 10.2140/pjm.1970.33.737.

[9] C.K. Fong, On the essential maximal numerical range, Acta Sci. Math., 41 (1979), 307315.

[10] L.A. Fialkow, Elementary Operators and Applications, In: Proceeding of the International Workshop, 1991.

[11] L. A. Fialkow, Spectral properties of elementary operators, Acta Sci. Math., 46 (1983), 269-282.

[12] C. Apostol and L. Fialkow, Structural properties of elementary operators, Can. J. Math., 38 (1986), 1485-1524, doi: 10.4153/CJM-1986-072-6.

[13] R.E. Curto and M. Mathieu, Elementary operators and their applications, In: 3-rd International Workshop, 2009, doi: 10.1007/978-3-0348-0037-2.

[14] Gerard J. Murphy, $C^{*}$-Algebras and Operator Theory, Academic Press Inc., Boston, MA, 1990.

[15] M. Takesaki, Theory of Operator Algebras I, Springer-Verlag, New York, 1979, doi: 10.1007/978-1-4612-6188-9.

[16] S. Sakai, $C^{*}$-Algebras and $W^{*}$-Algebras, Springer, Berlin, 1971, doi: 978-3-642-61993-9.

[17] O. Bratteli and D. W. Robinson, Operator Algebras and Quantum Statistical Mechanics, Volumes 1 and 2, Springer, Berlin, 1987 and 1997, doi: 10.1007/978-3-662-02520-8, and doi: 10.1007/978-3-662-03444-6. 
